\title{
EXISTENCE ET ÉQUIDISTRIBUTION DES MATRICES DE DÉNOMINATEUR $n$ DANS LES GROUPES UNITAIRES ET ORTHOGONAUX
}

\author{
par
}

Antonin Guilloux

\begin{abstract}
Let $\mathbf{G}$ be a simply-connected $\mathbb{Q}$ quasisimple and $\mathbb{R}$ anisotropic algebraic $\mathbb{Q}$-group. Let $\mathbb{A}^{f}$ be the finite part of the adèles $\mathbb{A}$ of $\mathbb{Q}$. Let $\left(H_{n}\right)$ be a sequence of bounded subsets of $\mathbf{G}\left(\mathbb{A}^{f}\right)$ which are bi-invariant by a compact open subgroup of $\mathbf{G}\left(\mathbb{A}^{f}\right)$. Let $\Gamma_{n}$ be the projection in $\mathbf{G}(\mathbb{R})$ of the sets $\mathbf{G}(\mathbb{Q}) \cap\left(\mathbf{G}(\mathbb{R}) \times H_{n}\right)$. Suppose that the volume of the compact subsets $\mathbf{G}(\mathbb{R}) \times H_{n}$ tends to $\infty$ with $n$. We prove the equidistribution in $\mathbf{G}(\mathbb{R})$ of the $\Gamma_{n}$ with respect to the Haar probability on $\mathbf{G}(\mathbb{R})$. The strategy is to use a mixing result for the action of $\mathbf{G}(\mathbb{A})$ on the space $L^{2}(\mathbf{G}(\mathbb{A}) / \mathbf{G}(\mathbb{Q}))$. As an application, we study the existence and the repartition of rational unitary matrices having a given denominator. We prove a local-global principle for this problem and the equirepartition of the sets of denominator $n$-matrices when they are not empty. Then we study the more complicated case of non simply-connected groups applying it to quadratic forms.
\end{abstract}

\section{Introduction}

La théorie des formes quadratiques définies positives à coefficients entiers répond de manière satisfaisante aux deux questions suivantes :

- À quelles conditions une forme quadratique donnée représente un entier $n$ (c'està-dire qu'il existe un vecteur entier de norme $\sqrt{n}$ ) ?

- Quand un entier $n$ est représenté, quelle est la répartition des vecteurs entiers sur l'ellipsoïde des vecteurs de norme $\sqrt{n}$ ?

Citons les résultats les plus simples, qui sont obtenus quand le rang de la forme quadratique est au moins 5 :

Théorème 1.1 (W. Tartakowsky [17, C. Pommerenke [15])

Soit $q$ une forme quadratique définie positive de rang $k \geq 5$ à coefficients entiers. Alors il existe un entier $N_{0}$ tel que pour tout $n \geq N_{0}$, on a l'équivalence entre les deux assertions suivantes :

1. Pour tout nombre premier $p$, l'entier $n$ appartient à $q\left(\mathbb{Z}_{p}^{k}\right)$.

2. L'entier $n$ appartient à $q\left(\mathbb{Z}^{k}\right)$. 
De plus, l'ensemble des vecteurs $v$ de $\mathbb{Z}^{k}$ vérifiant $q(v)=n$ s'équirépartit sur l'ellipsoïde $q(x)=n$ quand $n$ tend vers l'infini.

Nous reviendrons dans la partie 5 sur ce théorème et sur le cas des formes de petit rang. Nous nous intéressons dans ce texte à un analogue dans le cadre des groupes unitaires ou orthogonaux de ce résultat.

1.1. Matrices de dénominateur $n$ dans le groupe unitaire. - Présentons maintenant nos résultats dans le cas unitaire (pour le cas orthogonal, on renvoie à nouveau à la partie 5). Pour tout entier $k \geq 2$ et tout anneau $A$, on note $\mathrm{M}(k, A)$ l'ensemble des matrices carrées de taille $k \times k$ à coefficients dans $A$. Définissons le dénominateur d'une matrice à coefficients dans $\mathbb{Q}[i]$ :

Définition 1.2. - Soient $k$ un entier et $A$ une matrice de $M(k, \mathbb{Q}[i])$.

Le dénominateur $d$ de $A$ est défini comme le plus petit entier $d \in \mathbb{N}^{*}$ tel que $d A$ soit une matrice de $\mathrm{M}(k, \mathbb{Z}[i])$.

On fixe $k \geq 2$. Soient $H \in \mathrm{M}(k, \mathbb{Z}[i])$ une matrice hermitienne définie positive, $h$ la forme hermitienne associée. Nous voulons comprendre le comportement de l'ensemble des matrices de $\mathrm{SU}(h, \mathbb{Q})$ de dénominateur $n$ : à quelles conditions cet ensemble est non vide, et dans ce cas, quelle est sa répartition dans le groupe $\mathrm{SU}(h, \mathbb{R})$. On peut reformuler le problème de la façon suivante : $A$ désigne un des anneaux $\mathbb{Z}$ ou $\mathbb{Z}_{p}$ (pour $p$ premier), et $A_{i}=\mathbb{Z}[i] \otimes_{\mathbb{Z}} A$. Pour tout entier $n$, on note $\mathcal{T}(n, H, A)$ l'ensemble des matrices $M \in \mathrm{M}\left(k, A_{i}\right)$ de déterminant $n^{k}$ telles que :

- les coefficients de $M$ sont premiers entre eux,

- la matrice $M$ est solution de l'équation $\left(E_{n}\right): M^{*} H M=n^{2} H$.

Dans le cas $A=\mathbb{Z}$ et pour tout entier $n$, une matrice $M$ est dans $\mathcal{T}(n, H, \mathbb{Z})$ si et seulement si la matrice $\frac{1}{n} M$ est un élément de $\mathrm{SU}(h, \mathbb{Q})$ de dénominateur $n$. De même dans le cas $A=\mathbb{Z}_{p}$, une matrice $M$ est dans $\mathcal{T}\left(n, H, \mathbb{Z}_{p}\right)$ si et seulement si la matrice $\frac{1}{n} M$ est un élément de $\mathrm{SU}\left(h, \mathbb{Q}_{p}\right)$ tel que le supremum de la norme $p$-adique des coefficients soit la norme $p$-adique de $\frac{1}{n}$.

On note enfin $\mathcal{U}(H, A)$ l'ensemble des $n \in \mathbb{Z}$ tels qu'il existe $M \in \mathcal{T}(n, H, A)$, et $\mathcal{U}_{l}(H)=\bigcap_{p \text { premier }} \mathcal{U}\left(H, \mathbb{Z}_{p}\right)$. Bien sûr, pour qu'il existe des matrices de dénominateur $n$ dans $\mathrm{SU}(h, \mathbb{Q})$, il faut que $n$ soit dans $\mathcal{U}(H, \mathbb{Z})$, et donc il faut que $n$ soit dans $\mathcal{U}_{l}(H)$.

Le théorème suivant assure que pour $n$ suffisamment grand, c'est la seule condition :

Théorème 1.3. - Soient $k \geq 2, H \in M(k, \mathbb{Z}[i])$ une matrice hermitienne définie positive. Alors il existe $N_{0} \in \mathbb{N}$ tel que pour tout $n \geq N_{0}$, les deux assertions suivantes sont équivalentes :

1. L'entier $n$ appartient à $\mathcal{U}_{l}(H)$.

2. L'entier $n$ appartient à $\mathcal{U}(H, \mathbb{Z})$.

1.2. Équirépartition des matrices rationnelles. - La méthode pour prouver ce théorème est de prouver un résultat plus fort, à savoir l'équirépartition dans $\mathrm{SU}(h, \mathbb{R})$ de l'ensemble $\Gamma_{n}$ des matrices de dénominateur $n$, quand $n$ tend vers l'infini dans $\mathcal{U}_{l}(H)$. Voici l'énoncé : 
Théorème 1.4. - Soient $k \geq 2, H \in M(k, \mathbb{Z}[i])$ une matrice hermitienne définie positive et $h$ la forme hermitienne associée. Notons $\mu$ la probabilité de Haar sur $S U(h, \mathbb{R})$. Pour tout entier $n$, soit $\Gamma_{n}$ l'ensemble des $\frac{1}{n} M$ pour $M \in \mathcal{T}(n, H, \mathbb{Z})$.

Alors quand $n$ tend vers l'infini dans $\mathcal{U}_{l}(H)$, les $\Gamma_{n}$ s'équirépartissent dans $S U(h, \mathbb{R})$, c'est-à-dire qu'on la convergence :

$$
\frac{1}{\operatorname{Card}\left(\Gamma_{n}\right)} \sum_{\gamma \in \Gamma_{n}} \delta_{\gamma} \underset{n \in \mathcal{U}_{l}(H)}{\stackrel{n \rightarrow \infty}{\longrightarrow}} \mu
$$

dans l'espace des probabilités sur $S U(h, \mathbb{R})$ muni de la topologie faible-*.

Remarque 1.5. - Pour vérifier la condition $n \in \mathcal{U}_{l}(H)$, il suffit de vérifier que l'ensemble $\mathcal{T}\left(n, H, \mathbb{Z}_{p}\right)$ est non vide pour les nombres premiers $p$ divisant $n$. En effet, si $p$ ne divise pas $n$, la matrice $n \cdot I d$ appartient à $\mathcal{T}\left(n, H, \mathbb{Z}_{p}\right)$.

La question de la répartition des points rationnels de dénominateur $n$ dans le groupe des points réels d'un groupe algébrique $\mathbf{G}$ défini sur $\mathbb{Q}$ quand $n$ tend vers l'infini a déjà été étudiée par plusieurs auteurs.

Dans le cas où $\mathbf{G}(\mathbb{R})$ est non-compact, A. Eskin et H. Oh $[\mathbf{9}$ ] ont démontré que ces points étaient équidistribués suivant la mesure de Haar de $\mathbf{G}(\mathbb{R})$. Pour cela ils utilisent la présence de sous-groupes unipotents dans $\mathbf{G}(\mathbb{R})$ et concluent grâce à des théorèmes de Ratner et Dani-Margulis. Cependant, dans le cas où $\mathbf{G}(\mathbb{R})$ est compact, il n'y a pas d'élément unipotent dans $\mathbf{G}(\mathbb{R})$, donc on ne peut pas appliquer ces théorèmes.

Une autre méthode pour prouver des théorèmes d'équirépartition est d'utiliser le mélange. On renvoie à l'article d'A. Eskin et C. McMullen 8 pour une présentation très claire de cette méthode. Pour pouvoir l'utiliser dans notre cas, il faut disposer d'un résultat de décroissance des coefficients de l'action de $\mathbf{G}$ sur $L^{2}(\mathbf{G}(\mathbb{A}) / \mathbf{G}(\mathbb{Q}))$ (dans ce cadre $\mathbf{G}(\mathbb{Q})$ est un réseau du groupe des points sur les adèles $\mathbf{G}(\mathbb{A})$ ). De tels résultats sont prouvés dans l'article de L. Clozel, H. Oh et E. Ullmo [5], et complétés dans un article de L. Clozel 4, puis de A. Gorodnik, F. Maucourant et H. Oh 11 où une décroissance des coefficients de l'action de $\mathbf{G}$ sur $L^{2}(\mathbf{G}(\mathbb{A}) / \mathbf{G}(\mathbb{Q}))$ est prouvée sous les hypothèses que $\mathbf{G}$ est un groupe algébrique défini sur un corps de nombres, connexe et absolument presque-simple (on renvoie au théorème 2.2 pour l'énoncé exact).

C'est ce dernier résultat que nous utiliserons. Commençons par rappeler quelques résultats sur les groupes algébriques et leurs réseaux arithmétiques, ce qui permettra de fixer les notations.

1.3. Notations. - Nous définissons dans cette partie les notations dont nous nous servirons dans ce texte. Il nous faudra pour cela faire appel à des résultats sur les groupes algébriques et adéliques. Pour leur preuve, nous renvoyons le lecteur d'une part à l'article [18 de $\mathrm{J}$. Tits (et ses références) pour les résultats spécifiques aux points sur $\mathbb{Q}_{p}$ d'un groupe algébrique, et d'autre part au livre de V. Platonov et A. Rapinchuk [14] pour les propriétés adéliques.

Fixons une fois pour toutes un groupe $\mathbf{G}$ défini sur un corps de nombres $K$, connexe, presque- $K$-simple (c'est-à-dire que tout sous- $K$-groupe distingué de $\mathbf{G}$ est fini). Soit 
$\mathcal{V}$ l'ensemble des places de $K$. On note $\mathbb{A}$ (resp. $\mathbb{A}^{f}$, resp. $\mathbb{A}^{\infty}$ ) l'anneau des adèles de $K$ (resp. des adèles finies, resp. infinies). On note de plus

$$
G=\mathbf{G}(\mathbb{A}) ; G^{f}=\mathbf{G}\left(\mathbb{A}^{f}\right) \text { et } G^{\infty}=\mathbf{G}\left(\mathbb{A}^{\infty}\right) .
$$

On supposera toujours que $G^{\infty}$ est compact.

Nous disposons alors du sous-groupe $\mathbf{G}(K)$. On rappelle que c'est un réseau de $G$, qu'il est irréductible car $\mathbf{G}$ est presque- $K$-simple; et enfin qu'il est cocompact car $\mathbf{G}$ est $K$-anisotrope.

On appelle réseau arithmétique de $G$ tout sous-groupe $\Gamma$ tel que $\Gamma \cap \mathbf{G}(K)$ est d'indice fini dans $\Gamma$ et dans $\mathbf{G}(K)$. D'après ce qui précède, tout réseau arithmétique $\Gamma$ de $G$ est irréductible et cocompact. On se fixe un tel réseau $\Gamma$, ainsi qu'un sous-groupe compact ouvert $U$ de $G^{f}$.

On dira qu'une suite d'éléments $\left(g_{n}\right)$ de $G$ tend vers l'infini si pour toute partie compacte $C$ de $G$, pour $n$ suffisamment grand, $g_{n}$ n'appartient pas à $C$.

On note $\tau^{\infty}$ la projection de $G \operatorname{sur} G^{\infty}, \tau^{f}$ la projection de $G$ sur $G^{f}$, et enfin $\pi$ la projection de $G$ sur $G / \Gamma$. De plus on note $\lambda$ la mesure de Haar sur $G^{f}$ normalisée par $\lambda(U)=1 ; \mu$ la probabilité de Haar sur $G^{\infty}$. On note enfin $m$ la probabilité sur $G / \Gamma$ localement proportionnelle à $\mu \otimes \lambda$ et on l'appelle probabilité de Haar sur $G / \Gamma$. Le diagramme ci-dessous résume ces données :

$$
\begin{aligned}
& G, \mu \otimes \lambda \\
& \tau^{\infty} \swarrow \quad \tau^{f} \downarrow \quad \searrow \pi \\
& G^{\infty}=G^{f} \backslash G, \mu \quad G^{f}, \lambda \quad G / \Gamma, m
\end{aligned}
$$

Nous utilisons les notations suivantes pour les fonctions caractéristiques et les masses de Dirac dans un ensemble $A$ : si $a$ est un élément de $A$, on note $\delta_{a}$ la masse de Dirac en $a$; si $B$ est une partie de $A$, on note $\mathbf{1}_{B}$ la fonction caractéristique de $B$.

Enfin, pour les applications, on supposera toujours fixée une base sur $\mathbb{Z}^{k}$ et $\mathbb{Z}[i]^{k}$. Ainsi, nous supposons fixée une fois pour toutes l'identification entre formes quadratiques (resp. hermitiennes) et matrices symétriques (resp. hermitiennes).

1.4. Application de la décroissance des coefficients. - On remarque qu'une matrice est de dénominateur $n$ si et seulement si pour tout nombre premier $p$, le maximum de la norme $p$-adique de ses coefficients est la norme $p$-adique de $\frac{1}{n}$. Donc on peut réénoncer notre problème comme un problème de répartition dans $G^{\infty}$ de sous-ensembles de $\Gamma$ définis par certaines conditions sur leur projection dans $G^{f}$. C'est dorénavant sous cet angle que nous travaillerons.

Nous prouvons dans ce cadre le résultat d'équirépartition suivant (rappelons que $U$ est un sous-groupe compact ouvert fixé de $G^{f}$ ) : pour une suite d'ensembles $H_{n} \subset G^{f}$ bi- $U$-invariants, notons $\Gamma_{n}$ l'ensemble des points de $\Gamma$ dont la projection dans $G^{f}$ appartient à $H_{n}$. Alors, si le cardinal des $\Gamma_{n}$ tend vers l'infini, ils s'équirépartissent dans $G^{\infty}$ vers la mesure de Haar sur $G^{\infty}$.

C'est l'objet du théorème suivant (pour lequel on utilise les notations définies en 1.3) : 
Théorème 1.6. - Soit $\mathbf{G}$ un $K$-groupe, presque-K-simple, connexe tel que le complété aux places archimédiennes $G^{\infty}$ est compact. Soient $U$ un sous-groupe compact ouvert du groupe des adèles finies $G^{f}$ et $\left(H_{n}\right)$ une suite de sous-ensembles compacts bi-U-invariants de $G^{f}$. Soit $\Gamma$ un sous-groupe arithmétique du groupe des adèles $G$ et $\Gamma_{n}=\Gamma \cap\left(G^{\infty} \times H_{n}\right)$. Supposons que Card $\left(\Gamma_{n}\right)$ tende vers l'infini.

Alors, la projection de $\Gamma_{n}$ dans $G^{\infty}$ s'équirépartit dans $G^{\infty}$; c'est-à-dire qu'on a la convergence :

$$
\lim _{n \rightarrow \infty} \frac{1}{\operatorname{Card}\left(\Gamma_{n}\right)} \sum_{\gamma \in \Gamma_{n}} \delta_{\tau \infty(\gamma)}=\mu .
$$

dans l'espace des probabilités sur $G^{\infty}$.

Nous obtiendrons avec le théorème 3.1 un équivalent de $\operatorname{Card}\left(\Gamma_{n}\right)$. Ce théorème sera prouvé dans la partie 3. De plus, on notera toujours $G_{n}=G^{\infty} \times H_{n}$.

Remerciements : L'auteur tient à remercier Y. Benoist pour les nombreuses discussions et les nombreux conseils qu'il lui a prodigués. L'auteur remercie également le referee pour l'attention accordée à ce travail et les intéressantes remarques formulées.

\section{Décroissance des coefficients}

2.1. Le théorème de décroissance. - Nous présentons dans cette partie le théorème de A. Gorodnik, F. Maucourant et $\mathrm{H}$. Oh. Pour cela, il nous faut comprendre la représentation de $G$ dans l'espace $L^{2}(G / \Gamma)$.

On note $\langle$,$\rangle le produit scalaire canonique dans L^{2}(G / \Gamma)$ et $g . f$ l'action de $g \in G$ sur une fonction $f$ de $L^{2}(G / \Gamma)$, donnée par $(g . f)(x \Gamma)=f\left(g^{-1} x \Gamma\right)$. Considérons $\Lambda$ l'ensemble des caractères unitaires de $G$ triviaux sur $\Gamma$. Ils forment une base du sousespace vectoriel de $L^{2}(G / \Gamma)$ engendré par les sous-représentations de dimension 1 de $G$. Nous notons $L_{0}^{2}(G / \Gamma)$ l'orthogonal de ce sous-espace dans $L^{2}(G / \Gamma)$.

Remarque 2.1. - Dans 11, la représentation de $G$ considérée est la représentation $\lambda$ de $G$ dans $L^{2}(\Gamma \backslash G)$ donnée $\operatorname{par}(\lambda(g) \varphi)(\Gamma x)=\varphi(\Gamma x g)$.

Considérons l'isométrie $\Psi$ entre $L^{2}(G / \Gamma)$ et $L^{2}(\Gamma \backslash G)$ donnée par l'égalité : $\Psi(f)(\Gamma x)=f\left(x^{-1} \Gamma\right)$. Alors, on a pour tout $f$ dans $L^{2}(G / \Gamma)$ et $g$ dans $G$, $\lambda(g) \Psi(f)=\Psi(g . f)$. Cette égalité nous permet d'utiliser les résultats de [11] dans notre cas.

Nous pouvons maintenant citer le théorème de A. Gorodnik, F. Maucourant et H. Oh [11, Corollaire 1.20]:1

Théorème 2.2. - Soit $\mathbf{G}$ un groupe défini sur un corps de nombres $K$, connexe et absolument presque-simple. Alors pour toutes fonctions $f$ et $h$ de $L_{0}^{2}(G / \Gamma)$, on a :

$$
|\langle f, g . h\rangle| \stackrel{g \rightarrow \infty}{\longrightarrow} 0
$$

Remarquons que dans [1], le produit scalaire est majoré grâce à une fonction $\bar{\xi}$ construite de manière explicite. Nous n'utiliserons pas ici cette estimée. Comme ceci permet de simplifier la preuve, nous en donnons un aperçu dans la partie suivante. 
2.2. Cas des groupes unitaires et orthogonaux. - Nous voulons dans cette partie donner une idée de la preuve du théorème précédent dans un cas simple mais pertinent pour nos applications : G est le groupe $\mathrm{S} O(q)$ où $q$ est une forme quadratique définie positive rationelle en au moins 5 variables (la méthode qu'on présente fonctionne sans modification pour un groupe $\mathrm{SU}(h), h$ de rang au moins 4$)$. Précisément, nous prouvons la proposition suivante :

Proposition 2.3. - Soit $k \geq 5$ et soit $q$ une $\mathbb{Q}$-forme quadratique définie positive sur $\mathbb{Q}^{k}$. Pour toutes fonctions $f$ et $g$ de $L_{0}^{2}(S O(q, \mathbb{A}) / S O(q, \mathbb{Q}))$, on a :

$$
|\langle f, g . h\rangle| \underset{g \in S \rightarrow O(q, \mathbb{A})}{\stackrel{g \rightarrow \infty}{\longrightarrow}} 0
$$

La preuve que nous donnons reprend les idées de [11. Comme nous ne cherchons qu'une décroissance des coefficients et non pas une majoration, nous pouvons par endroits aller un peu plus vite, par exemple en utilisant le théorème de Howe-Moore [19. Théorème 10.1.4].

Démonstration. - Nous devons d'abord nous assurer d'un fait qui nous permettra d'utiliser les résultats de $\mathrm{H}$. Oh $\mathbf{1 3}$, et le théorème de Howe-Moore :

Fait 2.4. - Soit $p$ un nombre premier tel que le groupe $S O\left(q, \mathbb{Q}_{p}\right)$ est non-compact. Soit $S . O\left(q, \mathbb{Q}_{p}\right)^{+}$le sous-groupe de $S . O\left(q, \mathbb{Q}_{p}\right)$ engendré par les éléments unipotents.

Alors il n'existe pas de vecteurs $S \cdot \dot{O}\left(q, \mathbb{Q}_{p}\right)^{+}$-invariants dans l'espace $L_{0}^{2}(S O(q, \mathbb{A}) / S O(q, \mathbb{Q}))$.

Ce fait est un conséquence de l'approximation forte [14, Paragraphe 7.2]. Nous renvoyons pour sa démonstration au lemme 3.8 de [10] et à sa preuve.

Soit $U$ le sous-groupe compact $\prod_{p \text { premier }} \mathrm{SO}\left(q, \mathbb{Z}_{p}\right)$ de $\mathrm{S} O(q, \mathbb{A})$. Par définition, une fonction $f$ dans l'espace $L_{0}^{2}(\mathrm{SO}(q, \mathbb{A}) / \mathrm{SO}(q, \mathbb{Q}))$ est $U$-finie si l'espace vectoriel engendré par U.f est de dimension finie. C'est une conséquence classique du théorème de Peter-Weyl que l'ensemble des fonctions $U$-finies est dense dans $L_{0}^{2}(\mathrm{SO}(q, \mathbb{A}) / \mathrm{SO}(q, \mathbb{Q}))$. Donc il suffit de démontrer la proposition 2.3 pour de telles fonctions. Si $f$ est $U$-finie, on note $d(f)$ la dimension de l'espace engendré par $U . f$. Pour $p$ un nombre premier, on définit de même la notion de fonction $\mathrm{S} O\left(q, \mathbb{Z}_{p}\right)$-finie et, si $f$ est un fonction $\mathrm{S} O\left(q, \mathbb{Z}_{p}\right)$-finie, on note $d(f, p)$ la dimension de l'espace engendré par $\left(\mathrm{S} O\left(q, \mathbb{Z}_{p}\right) . f\right)$. Bien sûr, si $f$ est $U$-finie, elle est $\mathrm{S} O\left(q, \mathbb{Z}_{p}\right)$-finie. Dans ce cas, on a $d(f) \geq d(f, p)$.

L'hypothèse sur $k$ nous assure que, pour $p$ suffisamment grand, le groupe $\mathrm{SO}\left(q, \mathbb{Q}_{p}\right)$ est de rang au moins 2 et qu'on dispose d'une décomposition de Cartan $\operatorname{SO}\left(q, \mathbb{Q}_{p}\right)=$ $\mathrm{S} \mathrm{O}\left(q, \mathbb{Z}_{p}\right) A^{+} \mathrm{S} \mathrm{O}\left(q, \mathbb{Z}_{p}\right)$ où $A^{+}$est un sous-semigroupe d'un tore déployé maximal. En effet ceci est une conséquence de résultats de cohomologie galoisienne [14, Théorème 6.7] ; mais aussi de manière plus élémentaire du lemme 5.3 dont nous avons besoin dans la partie 5 Notons $\mathcal{P}_{2}$ l'ensemble des nombres premiers vérifiant ces deux propriétés, et $\mathcal{P}_{1}$ le complémentaire de $\mathcal{P}_{2}$ dans l'ensemble $\mathcal{V}$ des places de $\mathbb{Q}$.

Pour un groupe de rang 2 sur $\mathbb{Q}_{p}$, une majoration uniforme des coefficients de n'importe quelle représentation unitaire est donnée par les résultats de $\mathrm{H}$. Oh $\mathbf{1 3}$. 
Nous résumons ce que nous utilisons de $\mathbf{1 3}$ dans le fait suivant, où l'on considère la représentation unitaire de $\mathrm{SO}\left(q, \mathbb{Q}_{p}\right) \subset \mathrm{S} \mathrm{O}(q, \mathbb{A})$ dans $L_{0}^{2}(\mathrm{SO}(q, \mathbb{A}) / \mathrm{SO}(q, \mathbb{Q}))$ :

Fait 2.5 (Oh $[\mathbf{1 3})$. - Il existe pour tout $p$ dans $\mathcal{P}_{2}$ une fonction $\xi_{p}: S . O\left(q, \mathbb{Q}_{p}\right) \rightarrow$ ]0,1] vérifiant les conditions suivantes:

- pour tout $g_{p} \in S . O\left(q, \mathbb{Q}_{p}\right)$, on a $\xi_{p}\left(g_{p}\right) \stackrel{g_{p} \rightarrow \infty}{\longrightarrow} 0$.

- pour tout $g_{p} \in S . O\left(q, \mathbb{Q}_{p}\right)$, si $g_{p}$ n'appartient pas à $S O\left(q, \mathbb{Z}_{p}\right)$, on a $\xi_{p}\left(g_{p}\right) \leq \frac{2}{\sqrt{p}}$,

- pour toutes fonctions $f$ et $h$ de $L_{0}^{2}(S O(q, \mathbb{A}) / S O(q, \mathbb{Q}))$ qui sont $U$-finies, on a :

$$
\left\langle f, g_{p} . h\right\rangle \leq \sqrt{d(f, p) d(h, p)}\|f\|_{2}\|h\|_{2} \xi_{p}\left(g_{p}\right) .
$$

Ce fait nous permet de gérer les nombres premiers de $\mathcal{P}_{2}$. Pour gérer les autres, qui sont en nombre fini par hypothèse, nous pouvons faire appel au théorème de HoweMoore [19, Théorème 10.1.4] qui donne la décroissance des coefficients pour toute représentation d'un produit fini de groupes $p$-adiques simples sans vecteurs invariants par le sous-groupe engendré par les éléments unipotents. Nous énonçons une version ad-hoc de ce théorème :

Fait 2.6 (Howe-Moore [19]). - Soit $G_{\mathcal{P}_{1}}$ le groupe $\prod_{p \in \mathcal{P}_{1}} S . O\left(q, \mathbb{Q}_{p}\right)$. Alors pour tous sous-ensembles compacts $F$ et $H$ de $L_{0}^{2}(S O(q, \mathbb{A}) / S O(q, \mathbb{Q}))$, on a la convergence :

$$
\sup _{f \in F ; h \in H}|\langle f, g . h\rangle| \underset{g \in G_{\mathcal{P}_{1}}}{\stackrel{g \rightarrow \infty}{\longrightarrow}} 0
$$

Fixons maintenant $f$ et $h$ deux fonctions $U$-finies, normalisées pour que $\|f\|_{2}=$ $\|h\|_{2}=1$. Soit $\left(g_{n}=\left(g_{p, n}\right)_{p \in \mathcal{V}}\right)$ une suite d'éléments de $\mathrm{S} \mathrm{O}(q, \mathbb{A})$ qui tend vers l'infini. Pour tout $n$, on note :

$$
g_{n}^{1}=\left(g_{p, n}\right)_{p \in \mathcal{P}_{1}} \text { et } g_{n}^{2}=\left(g_{p, n}\right)_{p \in \mathcal{P}_{2}} .
$$

De plus, pour nombre premier $p$, on note $\overline{g_{p, n}}=\left(g_{q, n}\right)_{q \neq p}$. Quitte à partitionner la suite $\left(g_{n}\right)$ en sous-suites, on a l'alternative suivante :

1. La suite $\left(g_{n}^{2}\right)$ reste dans une partie compacte.

2. La suite $\left(g_{n}^{2}\right)$ tend vers l'infini.

Nous traitons ces deux cas séparément.

Cas 1) : On applique le théorème de Howe-Moore à la suite $\left(g_{n}^{1}\right)$ dans le groupe $G_{\mathcal{P}_{1}}$ et aux deux sous-ensembles compacts $\{f\}$ et $\left\{g_{n}^{2} . h\right.$ pour $\left.n \in \mathbb{N}\right\}$ de $L_{0}^{2}(\mathrm{~S} \mathrm{O}(q, \mathbb{A}) / \mathrm{SO}(q, \mathbb{Q}))$. On obtient alors que le coefficient $\left\langle f, g_{n} . h\right\rangle=\left\langle f, g_{n}^{1} \cdot\left(g_{n}^{2} . h\right)\right\rangle$ tend vers 0 avec $n$.

Cas 2) : On remarque que pour tout nombre premier $p$, l'action de $\operatorname{SO}\left(q, \mathbb{Z}_{p}\right)$ commute à celle de $\overline{g_{p, n}}$. On en déduit que le vecteur $\overline{g_{p, n}} . h$ est $\mathrm{S} \mathrm{O}\left(q, \mathbb{Z}_{p}\right)$-fini et on a :

$$
d\left(\left(\overline{g_{p, n}} . h\right), p\right)=d(h, p) \leq d(h) .
$$

On peut donc appliquer la troisième assertion du fait 2.5. Pour $p$ dans $\mathcal{P}_{2}$, on obtient :

$$
\left\langle f, g_{n} . h\right\rangle=\left\langle f, g_{p, n} \cdot\left(\overline{g_{p, n}} \cdot h\right)\right\rangle \leq \sqrt{d(f) d(h)} \xi_{p}\left(g_{p, n}\right) .
$$

On en déduit que le coefficient $\left\langle f, g_{n} . h\right\rangle$ est majoré par :

$$
\sqrt{d(f) d(h)} \inf _{p \in \mathcal{P}_{2}} \xi_{p}\left(g_{p, n}\right) \text {. }
$$


Or les deux premières assertions du fait 2.5 impliquent que ce minimum tend vers 0 quand $g_{n}^{2}$ tend vers l'infini. Ceci termine la preuve de la proposition.

2.3. Invariance par un sous-groupe compact ouvert. - Pour appliquer ce théorème, nous devons comprendre comment s'écrit une fonction dans la décomposition de $L^{2}(G / \Gamma)$ en $L_{0}^{2}(G / \Gamma)$ et son orthogonal. Or les fonctions que nous étudierons seront toutes invariantes par un sous-groupe compact ouvert. Nous fixons donc un tel sous-groupe $U$ compact ouvert.

Notons alors $\Lambda_{U}$ l'ensemble des caractères unitaires $U$-invariants de $\Lambda$ et $G_{U}$ l'intersection de tous les noyaux des caractères de $\Lambda_{U}$ :

$$
G_{U}=\cap_{\chi \in \Lambda_{U}} \operatorname{Ker} \chi \text {. }
$$

On dispose alors du lemme suivant, en partie tiré du lemme 4.1 de [1] :

Lemme 2.7. - 1. L'ensemble $U G^{\infty} \Gamma$ est inclus dans $G_{U}$.

2. Le groupe $G_{U}$ est d'indice fini $N_{U}$ dans $G$.

3. Si $f \in L^{2}(G / \Gamma)$ est définie sur $\pi\left(G_{U}\right)$ et est $U$-invariante, alors on a :

$$
f-\left(\int_{\pi\left(G_{U}\right)} f d m\right) \mathbf{1}_{\pi\left(G_{U}\right)} \in L_{0}^{2}(G / \Gamma)
$$

4. Si $f \in L^{2}(G / \Gamma)$ est définie sur $\pi\left(G_{U}\right)$ et est $U$-invariante, alors on a la convergence :

$$
\langle h . f, f\rangle \underset{h \in G_{U}}{\stackrel{h \rightarrow \infty}{\longrightarrow}}\left(\int_{G / \Gamma} f d m\right)^{2} .
$$

Démonstration. - Le premier point est une conséquence de la continuité des caractères, et du fait que $G^{\infty}$ est connexe car $\mathbf{G}$ est connexe et $\mathbb{R}$-anisotrope. On en déduit le deuxième car, d'après la théorie de la réduction (version adélique) [14, Théorème 5.1], l'ensemble $G^{\infty} U \backslash G / \Gamma$ est fini.

Pour le point 3 : soit $\chi \in \Lambda$. On veut calculer $\langle f, \chi\rangle$. Dans un premier temps, si $U$ n'est pas dans le noyau de $\chi$, alors ce produit scalaire est nul. Ensuite, si $\chi \in \Lambda_{U}$, alors $\langle f, \chi\rangle=\int_{\pi\left(G_{U}\right)} f \mathrm{~d} m$.

Pour le dernier point : soit $\bar{f}=f-\left(\int_{\pi\left(G_{U}\right)} f \mathrm{~d} m\right) \mathbf{1}_{\pi\left(G_{U}\right)}$. D'après le point 4 , on peut appliquer le théorème 2.2 à $\bar{f}$. On a alors : $\langle\bar{f}, h \cdot \bar{f}\rangle \stackrel{h \rightarrow \infty}{\longrightarrow} 0$. Or on vérifie aisément que quand $h \in G_{U}$, on a $\langle\bar{f}, h . \bar{f}\rangle=\int_{G / \Gamma} f(h x) f(x) \mathrm{d} m-\left(\int_{G / \Gamma} f \mathrm{~d} m\right)^{2}$.

Nous pouvons maintenant démontrer le théorème 1.6 .

\section{Dualité}

Nous allons en réalité démontrer un théorème plus précis que le théorème 1.6. En effet, dans les hypothèses de ce théorème, on avait besoin de supposer que $\operatorname{Cạd}\left(\Gamma_{n}\right)$ tend vers l'infini. Cette hypothèse est en pratique difficile à vérifier. Par exemple dans 
le cadre unitaire décrit dans l'introduction, il faudrait pour appliquer le théorème 1.6 connaître a priori un grand nombre de solutions entières de l'équation $\left(E_{n}\right)$.

3.1. Le théorème d'équidistribution. - Dans le théorème suivant, cette hypothèse est remplacée par l'hypothèse que les ensembles compacts $G_{n} \cap G_{U}$ sont deux à deux distincts. On remarque que cette hypothèse est a priori plus simple à vérifier, car nous n'avons plus besoin de trouver des solutions entières. Nous reviendrons là-dessus pour les applications dans les parties 4 et 5 .

Nous utilisons les notations définies dans la partie 1.3:

Théorème 3.1. - Soit $\mathbf{G}$ un $K$-groupe, presque-K-simple, connexe tel que le complété aux places archimédiennes $G^{\infty}$ est compact. Soient $U$ un sous-groupe compact ouvert du groupe des adèles finies $G^{f}$ et $\left(H_{n}\right)$ une suite de sous-ensembles compacts bi-U-invariants de $G^{f}$. Soit $\Gamma$ un sous-groupe arithmétique du groupe des adèles $G$ et $\Gamma_{n}=\Gamma \cap\left(G^{\infty} \times H_{n}\right)$. On suppose que les ensembles $G_{n} \cap G_{U}$ sont deux à deux distincts.

Alors, la projection de $\Gamma_{n}$ dans $G^{\infty}$ s'équirépartit dans $G^{\infty}$ :

$$
\lim _{n \rightarrow \infty} \frac{(\mu \otimes \lambda)(G / \Gamma)}{(\mu \otimes \lambda)\left(G_{n} \cap G_{U}\right)} \sum_{\gamma \in \Gamma_{n}} \delta_{\tau^{\infty}(\gamma)}=\mu ;
$$

en particulier, $\operatorname{Card}\left(\Gamma_{n}\right) \sim_{n \rightarrow \infty} \frac{(\mu \otimes \lambda)\left(G_{n} \cap G_{U}\right)}{(\mu \otimes \lambda)(G / \Gamma)}$

Remarque 3.2. - Nous pouvons supposer que le groupe $\mathbf{G}$ est en réalité absolument presque-simple. En effet, si G est presque- $K$-simple, il existe une extension finie $L$ de $K$ et $\mathbf{H}$ un $L$-groupe absolument presque-simple tels que $\mathbf{G}$ est défini comme la restriction des scalaires de $L$ à $K$ [1, 6.21.ii]. Mais alors, par définition de la restriction des scalaires [14, Section 2.1.2], les $K$-points de G s' identifient canoniquement aux $L$-points de $\mathbf{H}$, et le produit des complétés de $\mathbf{G}$ aux places archimédiennes de $K$, c'est-à-dire le groupe $G^{\infty}$, est isomorphe au produit $H^{\infty}$ des complétés de $\mathbf{H}$ aux places archimédiennes de $L$. Donc pour démontrer le théorème pour le groupe $\mathbf{G}$, il suffit de le montrer pour le groupe absolument presque-simple $\mathbf{H}$.

Dorénavant, nous supposons toujours le groupe $\mathbf{G}$ absolument presque-simple et en particulier il vérifie les hypothèses du théorème 2.2

Avant de prouver ce théorème, nous avons besoin de savoir que, dans les condition du théorème, le volume des ensembles $G_{n} \cap G_{U}$ tend vers l'infini. Le lemme suivant énonce ce résultat bien connu des spécialistes :

Lemme 3.3. - Soit $C_{n}$ une suite de parties compactes bi-U-invariantes 2 à 2 distinctes de $G^{f}$. Alors on a la limite $: \lim _{n \rightarrow \infty} \lambda\left(C_{n}\right)=+\infty$

Ce lemme est une conséquence des formules de dénombrement du volume d'une double-classe pour la décomposition de Cartan dans un groupe $p$-adique. On peut trouver de telles formules dans [2, paragraphe 1.5] ou bien [12, 7.3]. La formule que nous utilisons découle directement d'une formule de dénombrement pour le volume des doubles classes modulo un sous-groupe d'Iwahori dans un groupe $p$-adique [18, Paragraphe 3.3]. 
Cependant, comme nous n'avons pas de références précises et que la preuve de ce résultat nécessite l'introduction des objets classiques d'étude des groupes semi-simples $p$-adiques, nous renvoyons cette preuve à la partie 6 .

3.2. Preuve par dualité. — Nous prouvons maintenant le théorème 3.1

Démonstration du théorème 3.1 - Fixons une fonction $\varphi$ continue sur $G^{\infty}$. Nous voulons prouver la limite suivante :

$$
\lim _{n \rightarrow \infty} \frac{(\mu \otimes \lambda)(G / \Gamma)}{(\mu \otimes \lambda)\left(G_{n} \cap G_{U}\right)} \sum_{\gamma \in \Gamma_{n}} \varphi\left(\tau^{\infty}(\gamma)\right)=\int_{G^{\infty}} \varphi \mathrm{d} \mu .
$$

Pour cela, nous définissons la fonction $f$ sur $G$ en posant $f\left(g_{\infty}, g_{f}\right)=\varphi\left(g_{\infty}\right)$. Nous posons ensuite $F_{n}(g, h)=\sum_{\gamma \in \Gamma} f\left(g \gamma h^{-1}\right) \mathbf{1}_{G_{n}}\left(g \gamma h^{-1}\right)$.

Remarque 3.4. - L'introduction de ces fonctions $F_{n}$ est classique. Elles sont par exemple utilisées dans [11, Partie 5]. On peut remarquer que, au moins dans le cas où $\varphi$ est la fonction constante égale à 1 , ces fonctions $F_{n}$ sont exactement celles introduites dans [8, Partie 5]. En effet, nous voyons ici le groupe $G$ comme l'espace symétrique $(G \times G) / G$, où $G$ agit sur $G \times G$ par $g \cdot\left(g_{1}, g_{2}\right)=\left(g g_{1}, g_{2} g^{-1}\right)$.

Plus généralement, la preuve du théorème 3.1 est un avatar de la méthode présentée dans $[\mathbf{8}$.

On observe que pour tous $u_{1}, u_{2}$ dans $U$ et $\gamma_{1}, \gamma_{2}$ dans $\Gamma$, on a :

$$
F_{n}\left(u_{1} g \gamma_{1}, u_{2} h \gamma_{2}\right)=F_{n}(g, h) .
$$

(En effet, on peut modifier les parties non-archimédiennes sans modifier la valeur de $f$ ). Ainsi $F_{n}$ est une fonction continue bornée définie sur $(G / \Gamma)^{2}$, invariante par l'action à gauche de $U \times U$. De plus, on remarque - en notant $e$ l'élément neutre de $G$ - que :

$$
F_{n}(e, e)=\sum_{\gamma \in \Gamma_{n}} \varphi\left(\tau^{\infty}(\gamma)\right) .
$$

La fonction $\varphi$ est continue sur le groupe compact $G^{\infty}$, elle est donc uniformément continue. Ainsi, soient $\varepsilon>0$ et $U_{\varepsilon}$ un voisinage de l'identité dans $G^{\infty}$ tels que pour tout $u$ et $v \in U_{\varepsilon}$, pour tout $g \in G^{\infty}$, on a $|\varphi(u g v)-\varphi(g)| \leq \varepsilon$. Notons $\beta$ la fonction $\frac{1}{\mu\left(U_{\varepsilon}\right)} \mathbf{1}_{U_{\varepsilon}}$. On pose maintenant :

$$
\begin{gathered}
\bar{\alpha}\left(g_{\infty}, g_{f}\right)=\beta\left(g_{\infty}\right) \mathbf{1}_{U}\left(g_{f}\right) \\
\text { et } \alpha(g)=(\mu \otimes \lambda)(G / \Gamma) \sum_{\gamma \in \Gamma} \bar{\alpha}(g \gamma) .
\end{gathered}
$$

Par construction, $\bar{\alpha}$ est une fonction sur $G, U$-invariante de support $U_{\varepsilon} \times U \subset G_{U}$ et on a $\int_{G} \bar{\alpha} d(\mu \otimes \lambda)=1$. On en déduit que $\alpha$ est une fonction dans $L^{2}(G / \Gamma), U$ invariante, définie sur $\pi\left(G_{U}\right)$ et d'intégrale par rapport à $m$ égale à 1 .

Soit $(x, y) \in(G / \Gamma)^{2}$, tel que $\alpha(x) \alpha(y) \neq 0$. Alors $x$ et $y$ appartiennent à $\pi\left(U_{\varepsilon} \times U\right)$, c'est-à-dire qu'ils s'écrivent $x=u u_{\varepsilon} \Gamma$ et $y=v v_{\varepsilon} \Gamma$ avec $u_{\varepsilon}$ et $v_{\varepsilon}$ dans $U_{\varepsilon}$, et $u$ et $v$ 
dans $U$. Ainsi en utilisant l'égalité 1 et le fait qu'appartenir à $G_{n}$ ne dépend pas de la partie archimédienne d'un élément, on a :

$$
F_{n}(x, y)=F_{n}\left(u_{\varepsilon}, v_{\varepsilon}\right)=\sum_{\gamma \in \Gamma} \varphi\left(u_{\varepsilon} \gamma v_{\varepsilon}\right) \mathbf{1}_{G_{n}}(\gamma) .
$$

Par définition de $U_{\varepsilon}$, on a alors :

$$
\left|F_{n}(x, y)-F_{n}(e, e)\right| \leq \varepsilon \operatorname{Card}\left(\Gamma \cap G_{n}\right) .
$$

Cela implique :

$$
\left|F_{n}(e, e)-\int_{G / \Gamma} \int_{G / \Gamma} F_{n}(x, y) \alpha(x) \alpha(y) \mathrm{d} m(x) \mathrm{d} m(y)\right| \leq \varepsilon \operatorname{Card}\left(\Gamma \cap G_{n}\right) .
$$

Pour fixer les notations, fixons $X$ un relevé de $G / \Gamma$ dans $G$. On note $\tilde{\alpha}$ le relevé de $\alpha: \tilde{\alpha}=\alpha \circ \pi$, et on note $\tilde{m}=\frac{(\mu \otimes \lambda)}{(\mu \otimes \lambda)(X)}$. Enfin notons $I_{n, \varepsilon}$ l'intégrale :

$$
I_{n, \varepsilon}=\int_{G / \Gamma} \int_{G / \Gamma} F_{n}(x, y) \alpha(x) \alpha(y) \mathrm{d} m(x) \mathrm{d} m(y)
$$

On fait alors le calcul suivant :

$$
\begin{aligned}
I_{n, \varepsilon} & =\int_{X} \int_{X} F_{n}(x, y) \tilde{\alpha}(x) \tilde{\alpha}(y) \mathrm{d} \tilde{m}(x) \mathrm{d} \tilde{m}(y) \\
& =\int_{X} \int_{X} \sum_{\gamma \in \Gamma} f\left(x \gamma y^{-1}\right) \mathbf{1}_{G_{n}}\left(x \gamma y^{-1}\right) \tilde{\alpha}(x) \tilde{\alpha}(y) \mathrm{d} \tilde{m}(x) \mathrm{d} \tilde{m}(y)
\end{aligned}
$$

On fait pour tout $\gamma \in \Gamma$ le changement de variable $x \gamma=g$, ce qui permet d'obtenir :

$$
I_{n, \varepsilon}=\int_{G} \int_{X} f\left(g y^{-1}\right) \mathbf{1}_{G_{n}}\left(g y^{-1}\right) \tilde{\alpha}(g) \tilde{\alpha}(y) \mathrm{d} \tilde{m}(g) \mathrm{d} \tilde{m}(y)
$$

On fait maintenant le changement de variables $h=g y^{-1}$ pour tout $g \in G$. On obtient finalement :

$$
I_{n, \varepsilon}=\int_{G} f(h) \mathbf{1}_{G_{n}}(h) \int_{X} \tilde{\alpha}(h y) \tilde{\alpha}(y) \mathrm{d} \tilde{m}(y) \mathrm{d} \tilde{m}(h)
$$

Considérons le coefficient matriciel $\int_{X} \tilde{\alpha}(h y) \tilde{\alpha}(y) \mathrm{d} \tilde{m}(y)=\langle h . \alpha, \alpha\rangle$ de la représentation de $G$ dans $L^{2}(G / \Gamma)$.

On remarque tout d'abord que si $h$ n'appartient pas à $G_{U}$, et $x \in G / \Gamma$ appartient à $\pi\left(G_{U}\right)$, l'élément $h x$ de $G / \Gamma$ n'appartient pas à $\pi\left(G_{U}\right)$; comme le support de $\alpha$ est inclus dans $\pi\left(G_{U}\right)$, on a alors $(h . \alpha)(x)=\alpha(h x)=0$. Cela signifie que le coefficient $\langle h . \alpha, \alpha\rangle$ est nul dès que $h \notin G_{U}$. C'est ainsi qu'apparaîssent les ensembles $G_{n} \cap G_{U}$ :

$$
I_{n, \varepsilon}=\int_{G} f(h) \mathbf{1}_{G_{n} \cap G_{U}}(h)\langle h . \alpha, \alpha\rangle \mathrm{d} \tilde{m}(h)
$$

Ensuite, nous appliquons le théorème de décroissance à ce coefficient matriciel. On utilise à nouveau le fait que $\alpha$ est définie sur $\pi\left(G_{U}\right)$ et aussi qu'elle est $U$-invariante et d'intégrale 1. En effet, cela permet d'appliquer le point 4 du lemme 2.7 et on obtient :

$$
\langle h . \alpha, \alpha\rangle \underset{h \in G_{U}}{\stackrel{h \rightarrow \infty}{\longrightarrow}} 1 \text {. }
$$


Ainsi il existe une partie compacte $C$ de $G_{U}$, tel que pour tout $h \in G_{U}-C$, on a la majoration $|\langle h . \alpha, \alpha\rangle-1| \leq \varepsilon$. De plus, par définition de $f$, on a :

$$
\int_{G} f(h) \mathbf{1}_{G_{n} \cap G_{U}}(h) \mathrm{d} \tilde{m}(h)=\frac{(\mu \otimes \lambda)\left(G_{n} \cap G_{U}\right)}{(\mu \otimes \lambda)(G / \Gamma)} \int_{G^{\infty}} \varphi \mathrm{d} \mu
$$

Et alors, pour $n$ suffisamment grand, il existe une constante $A$ telle que :

$$
\left|I_{n, \varepsilon}-\frac{(\mu \otimes \lambda)\left(G_{n} \cap G_{U}\right)}{(\mu \otimes \lambda)(G / \Gamma)} \int_{G^{\infty}} \varphi \mathrm{d} \mu\right| \leq \varepsilon \frac{(\mu \otimes \lambda)\left(G_{n} \cap G_{U}\right)}{(\mu \otimes \lambda)(G / \Gamma)} \int_{G^{\infty}} \varphi \mathrm{d} \mu+A .
$$

On utilise maintenant le fait que le volume $(\mu \otimes \lambda)\left(G_{n} \cap G_{U}\right)$ tend vers l'infini (voir le lemme 3.3) pour obtenir :

$$
\left|I_{n, \varepsilon}-\frac{(\mu \otimes \lambda)\left(G_{n} \cap G_{U}\right)}{(\mu \otimes \lambda)(G / \Gamma)} \int_{G^{\infty}} \varphi \mathrm{d} \mu\right| \leq \varepsilon \frac{2(\mu \otimes \lambda)\left(G_{n} \cap G_{U}\right)}{(\mu \otimes \lambda)(G / \Gamma)} \int_{G^{\infty}} \varphi \mathrm{d} \mu .
$$

On en déduit que pour $n$ grand, on a l'inégalité :

$$
\begin{aligned}
\left|F_{n}(e, e)-\frac{(\mu \otimes \lambda)\left(G_{n} \cap G_{U}\right)}{(\mu \otimes \lambda)(G / \Gamma)} \int_{G^{\infty}} \varphi \mathrm{d} \mu\right| \leq & \\
& \left(\frac{2(\mu \otimes \lambda)\left(G_{n} \cap G_{U}\right)}{(\mu \otimes \lambda)(G / \Gamma)} \int_{G^{\infty}} \varphi \mathrm{d} \mu+\operatorname{Cạd}\left(\Gamma \cap G_{n}\right)\right) \varepsilon .
\end{aligned}
$$

C'est à dire qu'on a pour tout $\varepsilon>0$ :

$$
\begin{aligned}
& \limsup _{n \rightarrow \infty} \frac{(\mu \otimes \lambda)(G / \Gamma)}{(\mu \otimes \lambda)\left(G_{n} \cap G_{U}\right)}\left(F_{n}(e, e)-\varepsilon \operatorname{Card}\left(\Gamma \cap G_{n}\right)\right) \leq(1+2 \varepsilon) \int_{G^{\infty}} \varphi \mathrm{d} \mu \\
& \liminf _{n \rightarrow \infty} \frac{(\mu \otimes \lambda)(G / \Gamma)}{(\mu \otimes \lambda)\left(G_{n} \cap G_{U}\right)}\left(F_{n}(e, e)+\varepsilon \operatorname{Card}\left(\Gamma \cap G_{n}\right)\right) \geq(1-2 \varepsilon) \int_{G^{\infty}} \varphi \mathrm{d} \mu
\end{aligned}
$$

On conclut en deux étapes : tout d'abord, on applique les deux inégalités précédentes à $\varphi=1$, auquel cas $F_{n}(e, e)=\operatorname{Cạd}\left(\Gamma \cap G_{n}\right)$. On en déduit (en faisant tendre $\varepsilon$ vers 0$)$ que :

$$
\frac{(\mu \otimes \lambda)(G / \Gamma)}{(\mu \otimes \lambda)\left(G_{n} \cap G_{U}\right)} \operatorname{Card}\left(\Gamma \cap G_{n}\right) \stackrel{n \rightarrow \infty}{\longrightarrow} 1
$$

Enfin, on utilise ce résultat pour traiter le cas général. Pour tout $\varepsilon>0$, on a :

$$
\begin{aligned}
& \limsup _{n \rightarrow \infty} \frac{(\mu \otimes \lambda)(G / \Gamma)}{(\mu \otimes \lambda)\left(G_{n} \cap G_{U}\right)} F_{n}(e, e) \leq(1+2 \varepsilon) \int_{G^{\infty}} \varphi \mathrm{d} \mu+\varepsilon \\
& \liminf _{n \rightarrow \infty} \frac{(\mu \otimes \lambda)(G / \Gamma)}{(\mu \otimes \lambda)\left(G_{n} \cap G_{U}\right)} F_{n}(e, e) \geq(1-2 \varepsilon) \int_{G^{\infty}} \varphi \mathrm{d} \mu-\varepsilon
\end{aligned}
$$

On peut maintenant faire tendre $\varepsilon$ vers 0 pour obtenir la limite voulue. Ainsi, le théorème 3.1 est prouvé, et donc aussi le théorème 1.6. 


\section{Cas des groupes unitaires}

Nous prouvons dans cette partie le théorème 1.4 et donc le théorème 1.3. Nous reprenons les notations donnée dans l'introduction.

Démonstration du théorème 1.4 - On applique le théorème 3.1 dans le cas suivant : le groupe $\mathbf{G}$ est le $\mathbb{Q}$-groupe $\mathrm{SU}(h)$. On note qu'il vérifie bien les hypothèses du théorème et de plus qu'il est simplement connexe [14, Paragraphe 2.3.3]. On choisit $\Gamma=\mathrm{S} U(h, \mathbb{Q})$, et $U$ le produit pour $p$ premier des sous-groupes compacts ouverts $\mathrm{SU}\left(h, \mathbb{Z}_{p}\right)$.

Pour tout $p$ premier et $r$ entier, on note $L_{p^{r}}$ le sous-ensemble de $\mathbf{G}\left(\mathbb{Q}_{p}\right)$ composé des matrices telles que le supremum de la valeur absolue des coefficients est $p^{r}$. Enfin

pour tout $n$ entier avec $n=\prod_{p \text { premier }} p^{\nu_{p}(n)}$, on note $H_{n}=\prod_{p \text { premier }} L_{p^{\nu_{p}(n)}}$.

On remarque alors que un entier $n$ appartient à $\mathcal{U}_{l}(H)$ si et seulement si $H_{n}$ est non vide. De plus, pour toute matrice $M$ de $\mathrm{M}(h, \mathbb{Z}[i]), M$ est dans $\mathcal{T}(n, H, \mathbb{Z})$ si et seulement si $\frac{1}{n} M$ appartient à $\mathbf{G}(\mathbb{R}) \times H_{n}$. C'est à dire que les ensembles $\Gamma_{n}$ définis dans l'énoncé du théorème sont bien égaux à $\Gamma \cap G_{n}$.

Pour pouvoir appliquer le théorème 3.1 il ne reste plus qu'à prouver que les ensembles $G_{n} \cap G_{U}$ sont distincts. Or on remarque que, pour $n$ dans $\mathcal{U}_{l}(H)$, les $H_{n}$ sont disjoints donc distincts. Et il en est de même des $G_{n}$. Il suffit alors de vérifier que $G_{U}=G$. Le lemme suivant appliqué à $L=G_{U}$ permet de conclure :

Lemme 4.1. - Soit $\mathbf{G}$ un $\mathbb{Q}$-groupe, presque-(Q-simple et simplement connexe. Alors tout sous-groupe $L$ fermé normal, contenant $\mathbf{G}(\mathbb{Q})$ et d'indice fini dans $\mathbf{G}(\mathbb{A})$ est égal $\grave{a} \mathbf{G}(\mathbb{A})$.

Démonstration. - En effet, soit $p$ un nombre premier tel que $\mathbf{G}\left(\mathbb{Q}_{p}\right)$ est isotrope (un tel $p$ existe, c'est même le cas pour presque tous les $p$ [14. Théorème 6.7]). Alors, par hypothèse de simple connexité [14, Paragraphe 7.2], le groupe $\mathbf{G}\left(\mathbb{Q}_{p}\right)$ est engendré par ses éléments unipotents et notamment ne contient pas de sous-groupe d'indice fini différent de lui-même. Donc le groupe $L \cap \mathbf{G}\left(\mathbb{Q}_{p}\right)$ (ici, on a plongé de façon naturelle $\mathbf{G}\left(\mathbb{Q}_{p}\right)$ dans $\left.\mathbf{G}(\mathbb{A})\right)$ est égal à $\mathbf{G}\left(\mathbb{Q}_{p}\right)$.

Ensuite, par la propriété d'approximation forte [14, Théorème 7.12], le produit $\mathbf{G}\left(\mathbb{Q}_{p}\right) \mathbf{G}(\mathbb{Q})$ est dense dans $\mathbf{G}(\mathbb{A})$. Donc $L=\mathbf{G}(\mathbb{A})$.

Cela finit la preuve des théorèmes 1.4 et 1.3 .

\section{Cas des groupes orthogonaux}

Nous nous intéressons dans cette partie au cas des groupes orthogonaux. Rappelons tout d'abord que les groupes orthogonaux ne sont pas simplement connexes [14, Paragraphe 2.3.2, Proposition 2.14], donc le lemme 4.1 ne s'applique pas.

De fait, la question du passage du local au global pour les formes quadratiques à coefficients entiers a été beaucoup étudié et le théorème cité au début de ce texte donne une réponse satisfaisante dans les cas où le rang vaut au moins 5. Esquissons, dans les grandes lignes, la stratégie pour prouver ce théorème : 
On dit qu'une forme quadratique $q$ définie positive représente (resp. représente localement) un entier naturel $n$ si $n$ appartient à $q(\mathbb{Z})$ (resp. à $q\left(\mathbb{Z}_{p}\right)$ pour tout $p$ premier). Nous rappelons aussi la définition du genre d'une forme quadratique $q$. C'est l'ensemble des formes quadratiques équivalentes à $q$ à la fois sur $\mathbb{Q}$ et sur tous les $\mathbb{Z}_{p}$ :

Définition 5.1. - Soient $q$ et $q^{\prime}$ des formes quadratiques à coefficients entiers de rang $k$, associées respectivement aux matrices $Q$ et $Q^{\prime}$. On dit qu'elles sont dans le même genre si elles vérifient les propriétés suivantes :

- il existe $g \in \mathrm{GL}(k, \mathbb{Q})$ tel que $Q^{\prime}={ }^{t} g Q g$.

- pour tout nombre premier $p$, il existe un élément $g_{p} \in \mathrm{GL}\left(k, \mathbb{Z}_{p}\right)$ tel que $Q^{\prime}=$ ${ }^{t} g_{p} Q g_{p}$

On prouve alors que si un entier est représenté localement par une forme quadratique $q$, il existe une forme dans le genre de $q$ qui le représente [3, Chap. 9, Théorème 1.3]. Ensuite, on démontre, du moins quand le rang est au moins 5, que toutes les formes d'un même genre représentent les mêmes entiers suffisamment grands. Cette dernière étape ne fonctionne pas en toute généralité en rang 4, et pas du tout en rang 3 , où il faut introduire le concept de genre-spin. Nous ne décrirons pas davantage ces théories et renvoyons à l'article de W. Duke [6 pour une présentation historique de ce problème, ainsi qu'à l'article de W. Duke et R. Schulze-Pillot [7] pour l'analyse du cas de rang 3.

Présentons maintenant les résultats que nous obtenons : fixons une forme quadratique $q$ rationnelle définie positive de rang $k \geq 3$, de matrice associée $Q$. Pour tout entier $n$ et pour $A=\mathbb{Z}$ ou $\mathbb{Z}_{p}$, notons $\mathcal{S}(n, q, A)$ l'ensemble des matrices $M \in \mathrm{M}(k, A)$ de déterminant $n^{k}$ telles que $\frac{1}{n} M$ est de dénominateur $n$, c'est-à-dire :

- les coefficients de $M$ sont premiers entre eux,

- la matrice $M$ est solution de l'équation $\left(F_{n}\right):{ }^{t} M Q M=n^{2} Q$.

Notons $\mathcal{R}_{l}(q)$ l'ensemble des entiers $n$ tels que pour tout nombre premier $p$, $\mathcal{S}\left(n, q, \mathbb{Z}_{p}\right)$ est non vide. On notera de plus $\mathcal{R}_{\text {genre }}(q)$ l'ensemble des entiers $n$ tels qu'il existe une forme $q^{\prime}$ dans le genre de $q$ avec $\dot{\mathcal{S}}\left(n, q^{\prime}, \mathbb{Z}\right)$ non vide.

De la même manière que dans le cas unitaire, nous cherchons des matrices dans $\mathcal{S}(n, q, \mathbb{Z})$, et à comprendre l'image de cet ensemble dans $\mathrm{S} \mathrm{O}(q, \mathbb{R})$.

Dans le cas des formes de rang au moins 5, nous prouvons avec le théorème 5.2 que si $n$ est dans $\mathcal{R}_{l}(q)$ avec en plus la condition que $n$ est premier à un certain entier fixé, et que $n$ est suffisamment grand, alors $\mathcal{S}(n, q, \mathbb{Z})$ est non vide, et son image dans $\mathrm{S} O(q, \mathbb{R})$ s'équirépartit vers la mesure de Haar. Le concept de genre n'intervient pas dans cette partie.

Ensuite, nous essayons de suivre une stratégie parallèle à celle évoquée plus haut. Nous prouvons, cette fois sans restriction sur le rang, que, si $n$ est dans $\mathcal{R}_{\text {genre }}(q)$ et suffisamment grand, alors $\mathcal{S}(n, q, \mathbb{Z})$ est non vide, et son image s'équirépartit dans $\mathrm{SO}(q, \mathbb{R})$. C'est l'objet du théorème 5.7 .

5.1. Formes de rang supérieur à 5. - Commençons par le cas du rang supérieur à 5 : 
Théorème 5.2. - Soient $k \geq 5$, q une $\mathbb{Q}$-forme quadratique définie positive sur $\mathbb{Q}^{k}$ et $\mu$ la probabilité de Haar sur $S O(q, \mathbb{R})$. Pour tout entier $n$, notons $\Gamma_{n}$ l'ensemble des matrices de $S O(q, \mathbb{Q})$ de dénominateur $n$.

Alors il existe un entier $N$ tel que quand $n$ tend vers l'infini et que $n$ est premier à $N$, les $\Gamma_{n}$ s'équirépartissent dans $S O(q, \mathbb{R})$, c'est-à-dire :

$$
\frac{1}{\operatorname{Card}\left(\Gamma_{n}\right)} \sum_{\gamma \in \Gamma_{n}} \delta_{\gamma} \underset{n \text { premier } \grave{a} N}{\longrightarrow} \mu .
$$

Démonstration. - Nous allons à nouveau appliquer le théorème 3.1 en considérant le groupe $\mathbf{G}=\mathrm{S} \cdot \mathrm{O}(q)$, qui en vérifie bien les hypothèses. On pose pour tout nombre premier $p, U_{p}=\mathrm{S}, \mathrm{O}\left(q, \mathbb{Z}_{p}\right)$, et on note $U$ le produit sur $p$ des $U_{p}$.

Soit, pour $p$ premier et $m$ entier, $\tilde{H}_{p^{m}}$ l'ensemble des matrices de $\mathrm{SO}\left(q, \mathbb{Q}_{p}\right)$ telles que le maximum de la norme $p$-adique des coefficients est $p^{m}$. Maintenant, pour un entier $n$, pour tout nombre premier $p$, on note $\nu_{p}(n)$ la valuation $p$-adique de $n$. On pose alors $H_{n}$ le produit sur les $p$ premiers des $\tilde{H}_{p^{\nu p(n)}}$. Ces ensembles $H_{n}$ sont bi- $U$-invariants et deux à deux disjoints.

On vérifie alors que, pour tout $n$, l'ensemble $\Gamma_{n}$ est exactement $\mathrm{S} \mathrm{O}(q, \mathbb{Q}) \cap G_{n}$.

Il nous faut maintenant déterminer un ensemble fini $F$ de nombres premiers tel que si $n$ est premier aux éléments de $F$, alors $G_{n} \cap G_{U}$ est non vide. Il suffira alors de choisir pour $N$ le produit des nombres premiers dans $F$. Pour cela, on commence par un lemme de réduction des formes quadratiques :

Lemme 5.3. - Soient $k \geq 5, q$ une $\mathbb{Q}$-forme quadratique définie positive sur $\mathbb{Q}^{k}$. Alors il existe un ensemble fini $F$ de nombres premiers tels que pour tout nombre premier $p$ en dehors de $F$, on a:

la forme q est conjuguée par une matrice de $G L\left(k, \mathbb{Z}_{p}\right)$ à une forme quadratique de la forme $q^{\prime}\left(x_{1}, \ldots, x_{k}\right)=x_{1} x_{2}+x_{3} x_{4}+q^{\prime \prime}\left(x_{5}, \ldots, x_{k}\right)$.

Démonstration. - La forme $q$ est conjuguée par $A \in \mathrm{GL}(n, \mathbb{Q})$ à une forme quadratique diagonale $\bar{q}$. Soit $F$ l'ensemble des nombres premiers $p$ tels que ou bien $p=2$ ou bien $A$ n'appartient pas à $\mathrm{GL}\left(k, \mathbb{Z}_{p}\right)$ ou bien $\bar{q}$ n'est pas à coefficients dans $\mathbb{Z}_{p}^{*}$ pour la base canonique. Alors, pour tout $p \notin F, q$ est conjugué sur $\mathrm{GL}\left(k, \mathbb{Z}_{p}\right)$ à une forme quadratique diagonale à coefficients dans $\mathbb{Z}_{p}^{*}$.

Soit $r$ une forme quadratique diagonale en trois variables à coefficients dans $\mathbb{Z}_{p}^{*}$. L'ensemble $\mathbb{Z}_{p}^{*} /\left(\mathbb{Z}_{p}^{*}\right)^{2}$ est composé de deux éléments. Donc, $r$ est équivalente sur $\mathbb{Z}_{p}^{*}$ à $\alpha\left(x^{2}+y^{2}\right)+\beta z^{2}$, pour un $\alpha \in \mathbb{Z}_{p}^{*}$ et un $\beta \in \mathbb{Z}_{p}^{*}$. De plus tout élément de $\mathbb{Z}_{p}^{*}$ s'écrit comme somme de deux carrés par le lemme de Hensel [16, Chap II, section 2.2] (on utilise ici $p \neq 2)$, donc il existe $a$ et $b$ dans $\mathbb{Z}_{p}^{*}$ tels que $\alpha\left(a^{2}+b^{2}\right)=-\beta$. Alors, dans la base $\left((a, b, 1), \beta^{-1}(-b, a, 1),(a-b, a+b, 1)\right)$, la forme $r$ s'écrit $x y+\beta z^{2}$. Par construction la matrice qui conjugue $r$ à cette dernière forme est bien dans $\mathrm{GL}\left(k, \mathbb{Z}_{p}\right)$.

Pour la forme $q$ diagonale en au moins cinq variables, on peut appliquer le procédé ci-dessus deux fois pour obtenir le résultat voulu : pour tout nombre premier $p$ impair, toute forme quadratique $r$ diagonale sur $\mathbb{Q}_{p}^{5}$ à coefficients dans $\mathbb{Z}_{p}^{*}$ est équivalente sur $\mathbb{Z}_{p}$ à une forme quadratique $y_{1} y_{2}++y_{3} y_{4}+\alpha y_{5}^{2}$ pour un $\alpha \in \mathbb{Z}_{p}^{*}$. 
Pour tout $p \notin F$, on note $\varphi_{p}$ l'isomorphisme entre $\mathrm{S} \mathrm{O}\left(q, \mathbb{Q}_{p}\right)$ et $\mathrm{S} \mathrm{O}\left(q^{\prime}, \mathbb{Q}_{p}\right)$ donné par le lemme précédent et pour tout $m$ entier, $J_{p^{m}}$ l'ensemble des matrices de $\mathrm{SO}\left(q^{\prime}, \mathbb{Q}_{p}\right)$ telles que le maximum de la norme $p$-adique des coefficients est $p^{m}$. Comme le changement de base est à coefficients dans $\mathbb{Z}_{p}$, on obtient immédiatement le lemme :

Lemme 5.4. - Pour tout $p \notin F$, pour tout $m \in \mathbb{N}$, on a $\varphi_{p}\left(\tilde{H}_{p^{m}}\right)=J_{p^{m}}$.

Rappelons que $G_{U}$ est défini comme l'intersection des noyaux de l'ensemble $\Lambda_{U}$ des caractères $U$ et $\Gamma$-invariants de $G$. Soit $n$ un entier. On veut démontrer que $G_{n} \cap G_{U}$ est non vide. Supposons qu'on dispose de $g \in G$ et $u \in U$ tel que $g u g^{-1}$ soit un élément de $G_{n}$. Alors, pour tout $\lambda \in \Lambda^{U}, \lambda\left(g u g^{-1}\right)=1$, et donc $g u g^{-1} \in G_{n} \cap G_{U}$.

On note $N=\prod_{p \in F} p$. On va prouver que l'entier $N$ convient pour le théorème 5.2 : il suffit de démontrer que pour tout entier $n$ premier à $N$, on peut trouver une paire $(g, u) \in G \times U$ tel que $g u g^{-1}$ est dans $G_{n}$. Soit donc $n$ un entier premier à $N$.

D'après la définition de $H_{n}$, il suffit de trouver, pour tout $p$ premier et $m=\nu_{p}(n)$ entier, une paire $(g, u) \in \mathrm{SO}\left(q, \mathbb{Q}_{p}\right) \times U_{p}$ telle que $g u g^{-1}$ appartient à $\tilde{H}_{p^{m}}$. Si $m=0$, ce qui est le cas notamment si $p \in F$, il suffit de trouver une matrice dans $\operatorname{SO}\left(q, \mathbb{Z}_{p}\right)$ : la matrice identité convient. Il reste à traiter le cas $\nu_{p}(n) \neq 0$, pour lequel on sait que $p \notin F$. Donc on peut appliquer les deux lemmes précédents pour l'isomorphisme $\varphi_{p}$. Le théorème est alors une conséquence du lemme suivant :

Lemme 5.5. - Soient $p$ un nombre premier impair, $m \in \mathbb{N}, k \geq 5$ et $q^{\prime}$ une forme quadratique sur $\mathbb{Q}_{p}^{k}$ de la forme $q^{\prime}\left(x_{1}, \ldots, x_{k}\right)=x_{1} x_{2}+x_{3} x_{4}+q^{\prime \prime}\left(x_{5}, \ldots x_{k}\right)$.

Alors il existe $g \in S O\left(q^{\prime}, \mathbb{Q}_{p}\right)$, et $u \in S O\left(q^{\prime}, \mathbb{Z}_{p}\right)$ tel que gug ${ }^{-1}$ appartient à $J_{p^{m}}$.

Démonstration. - Il suffit de prendre les matrices :

$$
g=\left(\begin{array}{ccccc}
p^{m} & 0 & 0 & 0 & 0 \\
0 & p^{-m} & 0 & 0 & 0 \\
0 & 0 & 1 & 0 & 0 \\
0 & 0 & 0 & 1 & 0 \\
0 & 0 & 0 & 0 & I_{k-4}
\end{array}\right) \text { et } u=\left(\begin{array}{ccccc}
1 & 0 & 0 & 0 & 0 \\
0 & 1 & 1 & 0 & 0 \\
0 & 0 & 1 & 0 & 0 \\
-1 & 0 & 0 & 1 & 0 \\
0 & 0 & 0 & 0 & I_{k-4}
\end{array}\right)
$$

Le théorème 5.2 est donc bien prouvé.

De même que dans le cas unitaire, on obtient comme corollaire un résultat d'existence :

Corollaire 5.6. - Soient $k \geq 5, Q \in M(k, \mathbb{Q})$ une matrice symétrique, définie positive. Alors il existe deux entiers $N$ et $n_{0}$ tels qu'on a pour tout entier $n \geq n_{0}$ :

$L$ 'entier $n$ est premier à $N$ implique que $\mathcal{S}(n, Q, \mathbb{Z})$ est non vide. 
5.2. Lien avec le genre. - Voilà l'énoncé qui exprime que pour des formes dans le même genre, l'ensemble des dénominateurs de matrices rationnelles dans leur groupe orthogonal sont les mêmes, du moins pour des entiers suffisamment grands :

Théorème 5.7. - Soient $k \geq 3$, q et $q^{\prime}$ deux formes quadratiques définies positives du même genre.

Alors, pour $n$ suffisamment grand, s'il existe une matrice rationnelle de dénominateur $n$ dans $S . O\left(q^{\prime}, \mathbb{Q}\right)$, il en existe une dans $S O(q, \mathbb{Q})$.

Démonstration. - On se place exactement dans le cadre de la preuve du théorème 5.2 : on considère à nouveau le groupe $\mathbf{G}=\mathrm{SO}(q)$. On pose pour tout nombre premier $p, U_{p}=\mathrm{S} \mathrm{O}\left(q, \mathbb{Z}_{p}\right)$, et $U$ le produit sur $p$ des $U_{p}$.

On définit encore, pour $p$ premier et $m$ entier, $\tilde{H}_{p^{m}}$ l'ensemble des matrices de $\mathrm{SO}\left(q, \mathbb{Q}_{p}\right)$ telles que le maximum de la norme $p$-adique des coefficients est $p^{m}$. Maintenant, pour un entier $n$, pour tout nombre premier $p$, on note $\nu_{p}(n)$ la valuation $p$-adique de $n$. On pose alors $H_{n}$ le produit sur les $p$ premiers des $\tilde{H}_{p^{\nu p(n)}}$. Ces ensembles $H_{n}$ sont bi- $U$-invariants et deux à deux disjoints.

Soit maintenant $n$ un entier tel qu'il existe une matrice $\gamma$ de dénominateur $n$ dans $\mathrm{SO}\left(q^{\prime}, \mathbb{Q}\right)$. Pour prouver le théorème, selon la méthode déjà vue, il nous suffit de prouver qu'alors $G_{n} \cap G_{U}$ est non vide. On note $Q$ et $Q^{\prime}$ les matrices associées à $q$ et $q^{\prime}$. Soient $g_{\mathbb{Q}}$ la matrice rationnelle conjuguant $Q$ et $Q^{\prime}$ et, pour tout nombre premier $p$, $g_{p}$ la matrice de $\mathrm{GL}\left(k, \mathbb{Z}_{p}\right)$ conjuguant $Q$ et $Q^{\prime}$. On note $g$ l'élément $\left(g_{\mathbb{Q}},\left(g_{p}\right)_{p}\right.$ premier $)$ de $\mathrm{GL}(k, \mathbb{A})$ (ici, $g_{\mathbb{Q}}$ est vu comme un élément de $\left.\mathrm{GL}(k, \mathbb{Q}) \subset \mathrm{GL}(k, \mathbb{R})\right)$. Considérons l'élément $g \gamma g^{-1}$ de $\mathrm{GL}(k, \mathbb{A})$. Alors par définition de $g$, c'est un élément de $\mathrm{SO}(q, \mathbb{A})$. On veut démontrer qu'il est dans $G_{n} \cap G_{U}$.

Pour cela, commençons par remarquer que pour tout nombre premier $p, g_{p}$ est dans $\mathrm{GL}\left(k, \mathbb{Z}_{p}\right)$. Ainsi on ne change pas la norme $p$-adique de $\gamma$ en le conjuguant par $g_{p}$. Donc l'élément $g \gamma g^{-1}$ est bien dans $G_{n}$.

Soit ensuite $\lambda$ un caractère de $\Lambda_{U}$, c'est-à-dire $U$ et $\Gamma$-invariant. On veut démontrer que $\lambda\left(g \gamma g^{-1}\right)$ vaut 1 . Définissons sur $\operatorname{SO}\left(q^{\prime}, \mathbb{A}\right)$ le caractère $\lambda^{\prime}$ par : pour tout $h \in \mathrm{S} \mathrm{O}\left(q^{\prime}, \mathbb{A}\right), \lambda^{\prime}(h)=\lambda\left(g_{\mathbb{Q}} h g_{\mathbb{Q}}^{-1}\right)$ (ici $g_{\mathbb{Q}}$ est vu comme l'élément rationel de $\mathrm{GL}(k, \mathbb{A})$ dont chaque composant dans $\mathrm{GL}(k, \mathbb{R})$ et les $\mathrm{GL}\left(k, \mathbb{Q}_{p}\right)$ est la matrice $\left.g_{\mathbb{Q}}\right)$. Comme $g_{\mathbb{Q}}$ est une matrice rationnelle, $\lambda^{\prime}$ est $\mathrm{SO}\left(q^{\prime}, \mathbb{Q}\right)$-invariant. Or on a $\lambda\left(g \gamma g^{-1}\right)=\lambda^{\prime}\left(g_{\mathbb{Q}}^{-1} g \gamma g^{-1} g_{\mathbb{Q}}\right)$. De plus, par construction, $g^{-1} g_{\mathbb{Q}}$ est un élément de $\mathrm{S} \mathrm{O}\left(q^{\prime}, \mathbb{A}\right)$ et $\gamma$ est un élément de $\mathrm{S} \mathrm{O}\left(q^{\prime}, \mathbb{Q}\right)$. Donc, on a démontré le résultat voulu :

$$
\lambda\left(g \gamma g^{-1}\right)=\lambda^{\prime}\left(g_{\mathbb{Q}}^{-1} g \gamma g^{-1} g_{\mathbb{Q}}\right)=\lambda^{\prime}(\gamma)=1
$$

Cela termine la preuve : il suffit d'appliquer le théorème 3.1

5.3. Application à la forme canonique. - Dans cette section, on applique nos résultats au cas de la forme quadratique canonique. On note $q_{k}$ la forme quadratique canonique $x_{1}^{2}+\ldots+x_{k}^{2}$ sur $\mathbb{Z}^{k}$. La stratégie est la même, mais la différence notable est qu'on sait construire en rang 3 des matrices rationnelles de tout dénominateur impair dans $\mathrm{S} \bigcirc\left(q_{3}, \mathbb{Q}\right)$, donc nous n'avons plus de problème avec le groupe $G_{U}$. 
Corollaire 5.8. - Soient $k \geq 3$ et $\mu$ la probabilité de Haar sur $S . O\left(q_{k}, \mathbb{R}\right)$. Pour tout entier $n$, on note $\Gamma_{n}$ l'ensemble des matrices de $S O\left(q_{k}, \mathbb{Q}\right)$ de dénominateur $n$.

Alors quand $n$ tend vers l'infini et est impair, les $\Gamma_{n}$ s'équirépartissent dans $S O\left(q_{k}, \mathbb{R}\right)$, c'est-à-dire :

$$
\frac{1}{\operatorname{Card}\left(\Gamma_{n}\right)} \sum_{\gamma \in \Gamma_{n}} \delta_{\gamma} \underset{n \rightarrow \infty}{\stackrel{n \rightarrow \infty \text { impir }}{\longrightarrow}} \mu .
$$

Démonstration. - Commençons par le lemme suivant :

Lemme 5.9. - Pour tout entier impair $n$, il existe une matrice de dénominateur $n$ dans $S O\left(q_{k}, \mathbb{Q}\right)$.

Démonstration. - Il suffit de le faire pour $\mathrm{SO}\left(q_{3}, \mathbb{Q}\right)$. Soit alors $n$ un entier impair, et $x=a+i b+j c+k d$ un quaternion à coefficients entiers premiers entre eux de norme $n$.

Considérons la matrice de l'action de $x$ par conjugaison sur les quaternions purs. Elle s'écrit :

$$
\frac{1}{n}\left(\begin{array}{ccc}
a^{2}+b^{2}-c^{2}-d^{2} & 2(b c-a d) & 2(a c+b d) \\
2(a d+b c) & a^{2}-b^{2}+c^{2}-d^{2} & 2(c d-a b) \\
2(b d-a c) & 2(a b+c d) & a^{2}-b^{2}-c^{2}+d^{2}
\end{array}\right)
$$

On vérifie alors qu'elle est bien de dénominateur $n$ (il n'y a pas de simplification possible). De plus, par construction c'est une matrice de $\mathrm{S} \mathrm{O}\left(q_{3}, \mathbb{Q}\right)$.

On en déduit (avec les notations des preuves précédentes) que pour tout $n$ impair, $G_{n} \cap G_{U}$ est non vide, car il contient une matrice rationnelle. On en déduit immédiatement le résultat du corollaire, comme application du théorème 3.1 .

\section{Volume des doubles classes dans la décomposition de Cartan}

Nous donnons ici une preuve du lemme 3.3 . Soient donc $\mathbf{G}$ un groupe algébrique défini sur $K, U$ un sous-groupe compact ouvert de $G^{f}$ et $\lambda$ la mesure de Haar sur $G^{f}$ telle que $\lambda(U)=1$. On veut prouver que si $g$ tend vers l'infini dans $G^{f}, \lambda(U g U)$ tend aussi vers l'infini. Remarquons que localement ce résultat est bien connu et on sait exprimer le volume des doubles classes modulo un sous-groupe d'Iwahori très simplement en fonction de la longueur pondérée sur le groupe de Weyl affine. Cependant ici $U$ est un sous-groupe compact du groupe des adèles donc la projection de $U$ sur presque toutes les places est un sous-groupe compact maximal dans lequel un sousgroupe d'Iwahori est d'indice de plus en plus grand. Il nous faut donc contrôler ce phénomène. C'est l'objet du lemme6.2 qui ne présente pas de difficultés mais nécessite l'introduction du vocabulaire d'étude des groupes réductifs sur les corps locaux.

On remarque tout d'abord l'égalité :

$$
\lambda(U g U)=\operatorname{Cạd}(U g U / U)=\operatorname{Cạd}\left(U /\left(U \cap g U g^{-1}\right)\right) .
$$

De plus, on peut changer de sous-groupe compact ouvert en vertu du lemme suivant : 
Lemme 6.1. - Si $V \subset U$ est un autre sous-groupe compact ouvert de $G^{f}$, il existe une constante $c>1$ telle que pour tout $g$ dans $G$, on a :

$$
\frac{1}{c} \operatorname{Card}(V g V) \leq \operatorname{Card}(U g U) \leq c \operatorname{Card}(V g V) \text {. }
$$

Démonstration. - Soit $c$ l'indice $[U: V]$. D'une part, on a

$$
\text { Card } U g U / U \geq \text { Card } V g U / U=\text { Card } V /\left(V \cap g U g^{-1}\right) \text {; . }
$$

Comme $V$ est d'indice $c$ dans $U$, on a Card $U g U / U \geq \frac{1}{c}$ Card $V /\left(V \cap g V g^{-1}\right)$, ce qui prouve la première inégalité. D'autre part, on a :

$$
\text { Card } U g U / U=\text { Card } U /\left(U \cap g U g^{-1}\right) \leq \text { Card } U /\left(V \cap g V g^{-1}\right) \text {. }
$$

A nouveau, par définition de $c$, on a Card $U g U / U \leq c$ Card $V /\left(V \cap g V g^{-1}\right)$.

Notons $\Omega$ l'ensemble des places non-archimédiennes de $K$, fixons une place $\omega \in \Omega$ et raisonnons dans le groupe $\mathbf{G}\left(K_{\omega}\right)$. Le corps $K_{\omega}$ est une extension finie de $\mathbb{Q}_{p}$ pour un certain nombre premier $p$; on note $q$ le cardinal de son corps résiduel. Pour les résultats sur les groupes sur les corps locaux, nous nous référons à l'article de J. Tits (18), dont nous reprenons les notations.

Résumons les objets fournis par la théorie des groupes $p$-adiques dont nous aurons besoin : on dispose dans $\mathbf{G}\left(K_{\omega}\right)$ d'un tore maximal $K_{\omega}$-déployé $T_{\omega}$, et on note $N_{\omega}$ son normalisateur et $Z_{\omega}$ son centralisateur (ce sont des $K_{\omega}$-sous-groupes de $\mathbf{G}$ ).

De plus on définit les objets suivants :

1. Les groupes $X^{*}=\operatorname{Họm}_{K_{\omega}}\left(T_{\omega}\right.$, Mụlt) et $X_{*}=\operatorname{Họm}_{K_{\omega}}\left(\right.$ Mụltt, $\left.T_{\omega}\right)$ des caractères et co-caractères définis sur $K_{\omega}$ du tore, ainsi que $X^{*}(Z)=\operatorname{Họm}_{K_{\omega}}\left(Z_{\omega}\right.$, Mụlt).

2. L'espace vectoriel $V=\mathbb{R} \otimes X_{*}$, et le système de racines restreintes $\Phi \subset X^{*}$ associé au tore $T_{\omega}$.

3. Une application $\nu$ de $N_{\omega}\left(K_{\omega}\right)$ dans le groupe des transformations affines d'un espace $A$ sous $V$, définie en 1.2 de $\mathbf{1 8}$ comme l'unique extension de l'application de $Z_{\omega}\left(K_{\omega}\right)$ vérifiant (en notant $v_{\omega}$ la valuation associée à $\omega$ ) :

pour tous $z \in Z_{\omega}\left(K_{\omega}\right)$ et $\chi \in X^{*}(Z)$, on a $\chi(\nu(z))=v_{\omega}(\chi(z))$.

4. Le groupe de Weyl fini ${ }^{v} W=N_{\omega}\left(K_{\omega}\right) / Z_{\omega}\left(K_{\omega}\right)$ et le groupe $\tilde{W}=N_{\omega}\left(K_{\omega}\right) / \operatorname{ker}(\nu)$ qui contient le groupe de Weyl affine $W$ comme sous-groupe distingué d'indice fini. On identifie $\tilde{W}$ comme un sous-groupe des transformations affines de $V$ en choisissant dans $A$ un point spécial comme origine. ${ }^{v} W$ est alors l'ensemble des automorphismes de $\tilde{W}$ fixant l'origine.

5. Un choix d'un ensemble $\Phi^{+}$de racines positives dans $\Phi$, et donc une chambre $C$ contenant 0 dans $V$ définie comme l'ensemble des points $v$ de $V$ tels que pour tout $\chi \in \Phi^{+}$, on a $\chi(v) \geq 0$.

6. La chambre vectorielle $Y^{+}=\mathbb{R}^{+} \otimes C$ de $V$ et un sous-groupe compact ouvert $U_{\omega}$ de $\mathbf{G}\left(K_{\omega}\right)$ (le fixateur du point spécial) tels que $\mathbf{G}\left(K_{\omega}\right)$ est l'union des doubles classes $U_{\omega} a U_{\omega}$ pour $a \in Z_{\omega}^{+}=\nu^{-1}\left(Y^{+}\right)$(décompostion de Cartan)

De plus, si $n \in Z_{\omega}$ est tel que $\nu(n)$ est dans ${ }^{v} W$, alors $n$ appartient à $U_{\omega}$.

Enfin, on peut définir sur $\tilde{W}$ une fonction longueur pondérée à valeur entière (voir le paragraphe 3.3 de [18]) de la façon suivante : on note $\left(r_{i}\right)$ les symétries de $W$ 
associées à un système de racines simples dans $\Phi^{+}$. A chacun de ces éléments est associé un entier non nul $d\left(r_{i}\right)$. On écrit tout élément $w \in \tilde{W}$ sous la forme $w=$ $r_{i_{1}} \ldots r_{i_{l}} w_{0}$ où $w_{0}(C)=C$ et $r_{i_{1}} \ldots r_{i_{l}}$ est un mot réduit dans $W$. On pose alors $l(w)=d\left(r_{i_{1}}\right)+\ldots+d\left(r_{i_{l}}\right)$.

Alors, d'après la section 3.3 de $\mathbf{1 8}$ (voir aussi [12], 7.3), pour tout $a \in Z_{\omega}^{+}$, en notant $\nu(a)=w$, on a :

$$
\operatorname{Card}\left(U_{\omega} a U_{\omega} / U_{\omega}\right)=\frac{\sum_{y \in \in^{v} W w^{v} W} q^{l(y)}}{\sum_{y \in^{v} W} q^{l(y)}}
$$

On tire le résultat suivant de cette formule :

Lemme 6.2. -

$$
\text { 1. si } a \in Z_{\omega}^{+} \text {n'est pas dans } U_{\omega} \text {, on a : }
$$

$$
\operatorname{Card}\left(U_{\omega} a U_{\omega} / U_{\omega}\right) \geq p \text {. }
$$

2. si $a_{n}$ tend vers l'infini dans $Z_{\omega}^{+}$, on a $\operatorname{Card}\left(U_{\omega} a_{n} U_{\omega} / U_{\omega}\right) \rightarrow+\infty$.

Démonstration. - Commençons par le second point : si $a_{n}$ tend vers l'infini dans $\mathbb{Z}_{\omega}^{+}$, alors la longueur $l(\nu(a))$ aussi, ce qui suffit.

Pour le premier point : soit $a$ un élément de $Z_{\omega}^{+}$qui n'est pas dans $U_{\omega}$. Considérons $w_{0}$ le mot le plus court dans ${ }^{v} W \nu(a)$.

Si $w_{0}$ ne fixe pas $C$, alors $l\left(w_{0}\right) \geq 1$ et pour tout $w \in{ }^{v} W$, on a par définition $l\left(w w_{0}\right)=l(w)+l\left(w_{0}\right)$. On en déduit que Card $\left(U_{\omega} a U_{\omega} / U_{\omega}\right)$ est plus grand que $q^{l\left(w_{0}\right)}$, donc que $p$.

Si $w_{0}$ fixe $C$, alors $w_{0}$ n'est pas dans ${ }^{v} W$ (sinon $a$ appartient à $\left.U_{\omega}\right) \cdot w_{0}^{-1}$ envoie donc l'origine de $V$ sur un autre point $x$. Or il existe dans ${ }^{v} W$ une symétrie $s$ qui envoie $x$ sur un point n'appartenant pas à $C$. Alors, le point $w_{0} s w_{0}^{-1}$ est dans $W$ car $W$ est distingué dans $\tilde{W}$, mais pas dans ${ }^{v} W$, car l'origine est envoyée sur un point en dehors de $C$, donc n'est pas fixée.

Donc $w_{0} s$ s'écrit sous la forme $r w_{0}$, où $r$ est dans $W$ mais pas dans ${ }^{v} W$. En raisonnant comme précedemment, mais pour l'ensemble ${ }^{v} W r w_{0}$, on obtient aussi dans ce cas que $\operatorname{Card}\left(U_{\omega} a U_{\omega} / U_{\omega}\right)$ est plus grand que $p$.

Maintenant que nous disposons de tous ces objets, le lemme 3.3 peut être prouvé :

Preuve du lemme 3.3. - On fixe maintenant le compact ouvert $U_{0}=\prod_{\omega \in \Omega} U_{\omega}$. Considérons une suite $\left(g_{n}\right)$ d'élément de $G^{f}$ qui tend vers l'infini. Chaque $g_{n}$ s'écrit comme une suite $\left(g_{\omega, n}\right)_{\omega \in \Omega}$ dans le produit $\prod_{\omega \in \Omega} \mathbf{G}\left(K_{\omega}\right)$. On décompose toutes les coordonées dans la décomposition de Cartan : $g_{\omega, n} \in U_{\omega} a_{\omega, n} U_{\omega}$, avec $a_{\omega, n} \in Z_{\omega}^{+}$.

Soit $A$ un entier positif. Alors on veut prouver qu'il existe $N$ tel que pour tout $n \geq N, \lambda\left(U_{0} g_{n} U_{0}\right) \geq A$. Pour tout élément $g_{n}$ tel qu'il existe une place $\omega_{p}$ au dessus d'un nombre premier $p \geq A$ avec $a_{\omega_{p}, n} \notin U_{\omega_{p}}$, on a d'après le premier point du lemme 6.2 :

$$
\lambda\left(U_{0} g_{n} U_{0}\right)=\prod_{\omega \in \Omega} \operatorname{Card}\left(U_{\omega} a_{\omega, n} U_{\omega} / U_{\omega}\right) \geq
$$

$$
\operatorname{Card}\left(U_{\omega_{p}} a_{\omega_{p}, n} U_{\omega_{p}} / U_{\omega_{p}}\right) \geq p \geq A \text {. }
$$


Par ailleurs, considérons la sous-suite $\left(g_{n}\right)_{n \in S}$ telle que pour tout $n \in S$ et pour toute place $\omega$ au dessus d'un nombre premier $p \geq A$, l'élément $a_{\omega, n}$ appartient à $U_{\omega}$.

$\mathrm{Si}$ cette sous-suite est finie, le résultat voulu est prouvé. Sinon, comme $\left(g_{n}\right)$ sort de tout compact, il existe une place $\omega_{p}$ au dessus d'un nombre premier $p \leq A$ tel que la suite $\left(a_{\omega_{p}, n}\right)_{n \in S}$ tend vers l'infini dans $Z_{\omega_{p}}^{+}$. Alors, d'après le deuxième point du lemme 6.2, on a :

$\lambda\left(U_{0} g_{n} U_{0}\right)=\prod_{\omega \in \Omega} \operatorname{Card}\left(U_{\omega} a_{\omega, n} U_{\omega} / U_{\omega}\right) \geq$

$$
\operatorname{Card}\left(U_{\omega_{p}} a_{\omega_{p}, n} U_{\omega_{p}} / U_{\omega_{p}}\right) \stackrel{n \rightarrow \infty}{\longrightarrow}+\infty \text {. }
$$

Ainsi pour $n$ suffisamment grand, le volume $\lambda\left(U_{0} g_{n} U_{0}\right)$ est de toute façon supérieur à $A$. Cela prouve le résultat pour le groupe compact ouvert $U_{0}$. Or on a vu avec le lemme 6.1 que cela suffisait.

\section{Références}

[1] A. Borel \& J. Tits, « Groupes réductifs », Inst. Hautes Etudes Sci. Publ. Math. 27 (1965), p. 55-150.

[2] W. Casselman, Introduction to the theory of admissible representation of $p$-adic reductive groups, 1995, Disponible à l'adresse : http ://www.math.ubc.ca/ cass/research.html.

[3] J. Cassels, Rational quadratic forms, Academic Press, London, New York, San Francisco, 1978.

[4] L. Clozel, « Démonstration de la conjecture $\tau$ », Invent. Math. 151 (2003), p. 297-328.

[5] L. Clozel, H. OH \& E. Ullmo, « Hecke Operators and equidistribution of Hecke points », Invent. Math. 144 (2001), p. 327-351.

[6] W. Duke, « Some old problems and new results about quadratic forms », Notices A.M.S 44 (1997), p. 190-196.

[7] W. Duke \& R. Schulze-Pillot, « Representation of integers by positive ternary quadratic forms and equidistribution of lattice points on ellipsoids », Invent Math 99 (1990), p. $49-57$.

[8] A. Eskin \& C. McMullen, « Mixing, counting and equidistribution in Lie groups », Duke Math. J. 71 (1993), p. 181-209.

[9] A. Eskin \& H. OH, « Ergodic theoretic proof of equidistribution of Hecke points », Erg. The. and Dyn. Sys. (To appear).

[10] W. Gan \& H. Он, «Equidistribution of integer points on a family of homogeneous varieties : A problem of Linnik », Compos. Math. 136 (2003), p. 323-352.

[11] A. Gorodnik, F. Maucourant \& H. OH, « Manin's and Peyre's conjecture on rational points of bounded height and adelic mixing », Prépublication, disponible à l'adresse : http ://www.math.brown.edu/ heeoh/ (2006).

[12] B. Gross, «On the Satake isomorphism», in Galois Representations in Arithmetic Algebraic Geometry (R. T. A.J. Scholl, ed.), Cambridge University Press, 1998, p. 223-237.

[13] H. Он, « Uniform pointwise bounds for matrix coefficients of unitary representations and application to Kazhdan constants », Duke Math. J. 113 (2002), p. 133-192.

[14] V. Platonov \& A. Rapinchuk, Algebraic Groups and Number Theory, Academic Press, Boston MA, London, Sydney, 1994. 
[15] C. Pommerenke, « Über die Gleichverteilung von Gitterpunkten auf $m$-dimensionalen Ellipsoiden », Acta Arithmetica 5 (1959), p. 227-257.

[16] J. SerRe, Cours d'arithmétique, Presses Universitaires de France, Paris, 1995.

[17] W. TARtakowsky, « La détermination de la totalité des nombres représentables par une forme quadratique positive quaternaire », Compte Rendus de l'Académie des Sciences 186 (1928), p. 1684-1987.

[18] J. Tits, « Reductive Groups over Local Fields », Proceedings of Symposia in Pure Mathematics 33 (1979), p. 20-70.

[19] R. ZimmeR, Ergodic theory and semisimple groups, Birkhaüser, Boston, 1984.

4 septembre 2007

Antonin Guilloux, Département de Mathématiques et Applications, École Normale Supérieure, 45 rue d'Ulm, 75005 Paris, France. - E-mail : antonin.guilloux@ens.fr Url : http://www.dma.ens.fr/ aguillou/ 